\title{
Loss of constitutive activity of the growth hormone secretagogue receptor in familial short stature
}

\begin{abstract}
Jacques Pantel,1,2 Marie Legendre,1,2 Sylvie Cabrol,3,4,5 Latifa Hilal,6 Yassir Hajaji, ${ }^{6}$ Séverine Morisset,7,8 Sylvie Nivot, ${ }^{9}$ Marie-Pierre Vie-Luton, ${ }^{1,2}$ Dominique Grouselle, ${ }^{8,10}$ Marc de Kerdanet, ${ }^{11}$ Abdelkrim Kadiri, ${ }^{12}$ Jacques Epelbaum, ${ }^{8,10}$ Yves Le Bouc,,3,4,5 and Serge Amselem ${ }^{1,2}$

${ }^{1}$ INSERM, U654, Centre Hospitalier Universitaire Henri-Mondor, Créteil, France. ${ }^{2}$ Université Paris 12, Faculté de Médecine, Créteil, France. ${ }^{3}$ Assistance Publique-Hôpitaux de Paris, Hôpital Armand-Trousseau, Service d'Explorations Fonctionnelles Endocriniennes, Paris, France. ${ }^{4}$ INSERM, U515, Paris, France. ${ }^{5}$ Université Pierre et Marie Curie-Paris 6, Paris, France. ${ }^{6}$ Unité de Formation et de Recherche de Génétique et Biologie Moléculaire, Laboratoire de Génétique et Biologie Moléculaire de la Faculté des Sciences, Kenitra, Morocco. ${ }^{7}$ INSERM, U573, Paris, France. ${ }^{8}$ Université René Descartes, Centre Paul Broca, Paris, France. ${ }^{9}$ Centre Hospitalier Universitaire de Caen, Service de Pédiatrie A, Caen, France. ${ }^{10}$ INSERM, U549, Paris, France. ${ }^{11}$ Centre Hospitalier Universitaire de

Rennes, Service d'Endocrinologie Pédiatrique, Paris, France. ${ }^{12}$ Centre Hospitalier Universitaire Ibn Sina, Service d'Endocrinologie, Rabat, Morocco.
\end{abstract}

\begin{abstract}
The growth hormone (GH) secretagogue receptor (GHSR) was cloned as the target of a family of synthetic molecules endowed with GH release properties. As shown recently through in vitro means, this receptor displays a constitutive activity whose clinical relevance is unknown. Although pharmacological studies have demonstrated that its endogenous ligand - ghrelin - stimulates, through the GHSR, GH secretion and appetite, the physiological importance of the GHSR-dependent pathways remains an open question that gives rise to much controversy. We report the identification of a GHSR missense mutation that segregates with short stature within 2 unrelated families. This mutation, which results in decreased cell-surface expression of the receptor, selectively impairs the constitutive activity of the GHSR, while preserving its ability to respond to ghrelin. This first description, to our knowledge, of a functionally significant GHSR mutation, which unveils the critical importance of the GHSR-associated constitutive activity, discloses an unusual pathogenic mechanism of growth failure in humans.
\end{abstract}

\section{Introduction}

The growth hormone secretagogue receptor (GHSR) (1), an orphan 7-transmembrane $G$ protein-coupled receptor (GPCR), was cloned as the target of a family of synthetic molecules named growth hormone $(\mathrm{GH})$ secretagogues endowed with $\mathrm{GH}$ release properties (2). This receptor is highly expressed in the brain and in the pituitary (1). The first endogenous ligand of this receptor, discovered in 1999 and named ghrelin (3), is a hormone predominantly produced by the stomach (3) whose plasma levels fluctuate with food intake (4). As shown in rodents and in humans, ghrelin stimulates GH secretion $(3,5,6)$ and increases food intake and body weight (7-10). However, the study of genetically modified murine models in which ghrelin signaling was disrupted has provided a number of heterogeneous and sometimes conflicting findings regarding the effect of these targeted mutations on the GH/IGF-1 axis (11-14).

In the human, the phenotype of individuals with mutations in GHSR (MIM 601898) - if such defects indeed exist - is so far unknown. To this end, we screened the GHSR coding sequence for mutations in groups of individuals presenting with growth defects. The results obtained prompted us to investigate the biological properties associated with one particular mutated GHSR identified in several patients.

Nonstandard abbreviations used: CRE, cAMP-response element; GH, growth hormone; GHSR, GH secretagogue receptor; GPCR, G protein-coupled receptor; IGHD, isolated GH deficiency; ISS, idiopathic short stature; Luc, luciferase; SRE, serumresponse element.

Conflict of interest: The authors have declared that no conflict of interest exists. Citation for this article: J. Clin. Invest. 116:760-768 (2006). doi:10.1172/JCI25303.

\section{Results}

Identification of a GHSR mutation. Sequencing of the PCR products spanning the 2 GHSR coding exons and flanking intronic regions led to the identification of the same nucleotide variation in 2 unrelated patients belonging to the 2 groups defined in Methods. This substitution, a C-to-A transversion located within the first GHSR exon (c.611C $\rightarrow \mathrm{A})$, was found in the heterozygous state in a patient from the isolated GH deficiency (IGHD) group, whereas it was present in the homozygous state in a patient from the idiopathic short stature (ISS) group (Figure 1A and Table 1). Although the 2 patients were not known to be related, both of them originated from Morocco. We, therefore, tested whether this nucleotide variation, which generates a recognition site for the restriction enzyme $M n l I$, would represent a frequent polymorphism. To this end, we looked for this sequence variation in a control group of unrelated individuals of normal stature originating from Morocco $(n=100)$; as shown in Table 1, it was not observed in this population sample, a result indicating that it is not a frequent polymorphism. We also analyzed the coding sequence of the $G H-N$ and $G H R H-R$ genes in the 2 probands and found no gene abnormality. Conversely, none of the available DNA samples carrying mutations in the GH-N $(n=6)$ or in the GHRH-R $(n=1)$ gene was found to be mutated in GHSR.

This GHSR nucleotide substitution (p.A204E) predicts the replacement of an apolar and neutral residue (alanine) by a polar and charged amino acid (glutamate). At the protein level, this missense mutation is located in the second extracellular loop of the GHSR1a protein (Figure 1B) and involves a residue totally conserved in all species studied so far (Figure 1C). Such a high 
A

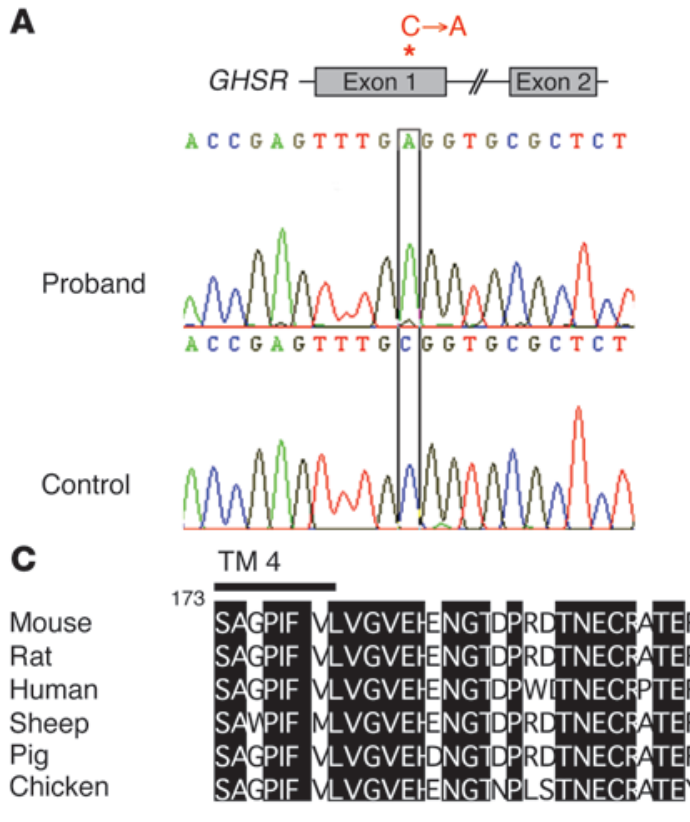

B

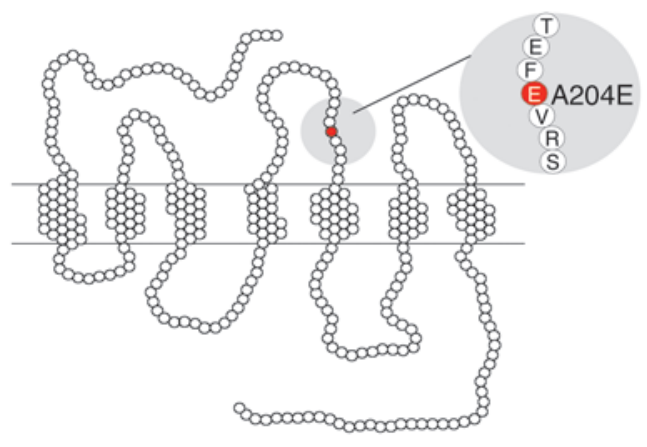

\section{Figure 1}

Identification of a GHSR mutation. (A) Electrophoregram spanning the GHSR mutation site from a proband presenting with ISS and a control. The sequence variation, present in the homozygous state, is a C-to-A transversion located within the first GHSR exon (c.611C $\rightarrow$ A). (B) Location of the predicted A204E mutation in the second extracellular loop of the GHSR1a, a 7-transmembrane G-coupled receptor. (C) Conservation of the GHSR1a amino acid sequence among species within the region bracketing the mutation site between the fourth (TM4) and the fifth (TM5) transmembrane domains; A204E is shown by an arrow. degree of conservation of alanine 204, therefore, suggests that amino acid substitutions at this position could be detrimental to receptor function.

Phenotype/genotype relationships. The segregation of this sequence variation with the short stature phenotype was subsequently analyzed within the 2 families. DNA samples of available individuals were amplified with the use of primers bracketing the mutation site, and the resulting PCR products were then digested by $\mathrm{Mnl \textrm {I }}$ (Figure 2). In family 1 (Figure $2 \mathrm{~A}$ ), in which the proband with ISS (individual II2) was found to carry the A204E mutation in the homozygous state, both parents (individuals I1 and I2) are heterozygous for this mutation, a result in keeping with the consanguinity documented in that family; this analysis revealed that all 3 siblings (individuals II1, II3, and II4) were also heterozygous for the mutation. Both parents have a significant height reduction: the father and the mother reached a final height of $152 \mathrm{~cm}$ and $148 \mathrm{~cm}$ (3.7 SD and 2.7 SD below the mean, respectively); the 2 heterozygous siblings II 3 and II 4 also have a reduced height (2.0 and 2.2 SD below the mean, respectively), whereas the last heterozygous sibling (II1) has a slight height reduction, albeit within the normal range (1.1 SD below the mean). In family 2 (Figure 2B), in which the proband with IGHD (individual II1) was found to carry the same mutation in the heterozygous state, the father, who has a slight height reduction (2.0 SD below the mean), is heterozygous, whereas 2 heterozygous siblings (II2 and III3) have a normal stature. Altogether, these data show that all individuals with a reduced height for age $(n=7)$ carry at least 1 A204E allele, in keeping with a dominant mode of inheritance of the short stature phenotype associated with this allele. Conversely, the 9 heterozygous individuals identified in this study do not all have a short stature, an observation that is consistent with incomplete penetrance of this phenotype; as 3 heterozygous individuals have a normal height, the penetrance of the short stature phenotype among heterozygous carriers is $66 \%$ in this sample.

Details of the clinical and biological phenotypes of the children with short stature from these 2 families are presented in Table 2, according to genotype. The sole homozygous patient (family 1-II2) has a phenotype compatible with the diagnosis of ISS, whereas heterozygous patients display phenotypic features compatible with the diagnosis of either ISS (family 1-II4) or IGHD (family 1-II3 and family 2-II1). Serum levels of IGF-1 were found to be low in the patient from family 2 (individual II1), whereas, in spite of the documented growth delay, they were found to be within the normal range in all 3 patients from family 1 (individuals II2, II3, and II4). Finally, among the children with short stature, the 3 who received a GH treatment increased their growth velocity (data not shown). The growth curve of the homozygous patient (family 1-II2) shows that, although her length was normal at birth $(50 \mathrm{~cm})$, a growth delay appeared within the first 2 years of life and worsened with age until the beginning of GH therapy (3.7 SD below the mean at the age of 13.8) (Figure 3). As for her weight, it remained within 2 SD below the normal mean for age until the age of 9 years and then increased continuously so that her BMI reached the overweight limit for age after the age of 13 years (Figure 3 and Table 2). Her parents, who carry the A204E mutation in the heterozygous state, are obese, whereas her heterozygous siblings display either a slight overweight (II1) or a trend to overweight (II3 and II4) (Table 2 and data not shown). In contrast, heterozygous individuals from family 2 have a BMI within the normal range, the proband (II1) even being rather lean.

Decreased cell-surface expression of GHSR1 a A204E. To evaluate the binding properties of ghrelin to the mutant GHSR1a, we performed ${ }^{125}$ I-ghrelin binding experiments on whole HEK293 cells transiently overexpressing the WT or the A204E mutant GHSR1a. Specific binding of ${ }^{125} \mathrm{I}$-ghrelin to the mutant recep-

\section{Table 1}

Distribution of the A204E GHSR sequence variation among unrelated patients with short stature and controls

\begin{tabular}{cccc} 
& ISS $(\boldsymbol{n}=\mathbf{4 1})$ & IGHD $(\boldsymbol{n}=\mathbf{5 1})$ & Controls $(\boldsymbol{n}=\mathbf{1 0 0})$ \\
A204E & 1 homozygote & 1 heterozygote & 0 \\
\hline
\end{tabular}


A

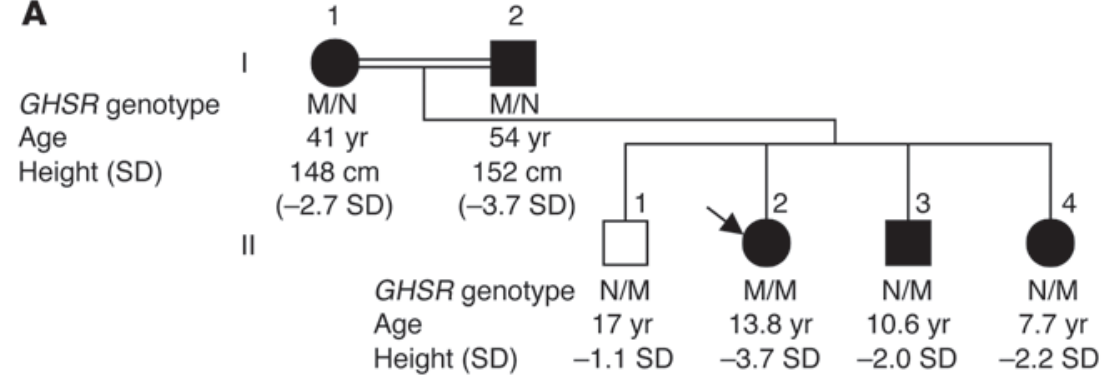

B

GHSR genotype
Age
Height (SD)

I

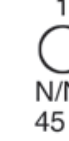

$158 \mathrm{~cm}$ $(-0.8 \mathrm{SD})$

II

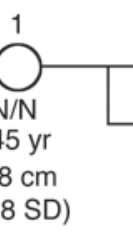

$\begin{array}{lccc}\text { GHSR genotype } & \mathrm{N} / \mathrm{M} & \mathrm{N} / \mathrm{M} & \mathrm{N} / \mathrm{M} \\ \text { Age } & 11.5 \mathrm{yr} & 11.3 \mathrm{yr} & 8.5 \mathrm{yr} \\ \text { Height (SD) } & -3.2 \mathrm{SD} & -1.2 \mathrm{SD} & +1.0 \mathrm{SD}\end{array}$
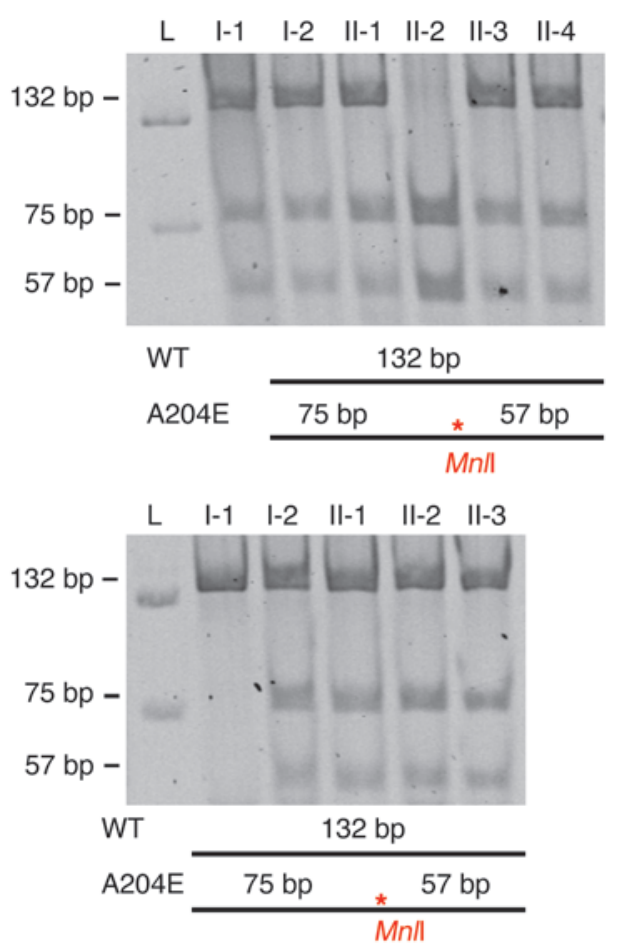

Figure 2

Inheritance of the A204E GHSR mutation in families 1 and 2. (A) Family 1. (B) Family 2. Circles and squares denote female and male family members, respectively. The SD to mean height for age is given below each symbol; height values are before GH treatment. Black symbols denote a short stature. The probands are indicated by arrows. The segregation of the GHSR A204E allele within both families was carried out by means of a specific restriction fragment length polymorphism (the A204E mutation creates an Mn/l site).

tor was extremely low, as compared with that associated with the WT GHSR1a (Figure 4A), in the presence of similar amounts of mutant and WT GHSR transcripts, as judged by means of semiquantitative RT-PCR analysis (data not shown). Finally, to assess the affinity of GHSR1a A204E for ${ }^{125} \mathrm{I}$-ghrelin - the low levels of binding to this mutant receptor in transient transfection experiments precluding any affinity measurements - we generated HEK293 cell lines stably expressing either the WT or the A204E receptor. As shown in Figure 4B, ${ }^{125} \mathrm{I}$-ghrelin displacement experiments performed on 2 representative cell lines (see Methods) showed very similar results $\left(\mathrm{IC}_{50}=2.38 \pm 0.54 \mathrm{nM}\right.$ and $1.68 \pm 0.22 \mathrm{nM}$ for the WT and the A204E receptor, respectively), therefore indicating that the A204E mutation has no significant effect on the affinity of the receptor for ghrelin.

To determine the subcellular localization of the mutant receptor, COS-7 cells transiently transfected with the WT or the HA-GHSR-A204E plasmid were examined by indirect immunofluorescence microscopy with an anti-HA tag antibody (Figure 4C). These experiments revealed a peripheral plasma membrane staining on nonpermeabilized cells expressing the WT or the mutant receptor, and after plasma membrane permeabilization, both transfectants exhibited intense cytoplasmic staining. Most importantly, the number of nonpermeabilized cells showing plasma membrane staining after transfection of the HA-GHSR-A204E plasmid was only $22 \%$ of the number showing plasma membrane staining after transfection of the HA-GHSRWT plasmid, a result further confirming the decreased cell-surface expression of the mutant receptor. Consistent with this hypothesis, the number of stained cells examined after transfection of HA-GHSR-A204E and plasma membrane permeabilization was slightly higher than that of cells expressing the WT plasmid. Overall, these observations support the fact that the A204E mutant is efficiently translated into a protein, but with only a small fraction properly expressed at the cell surface, this plasma membrane fraction displaying a normal affinity for ghrelin.

Loss of constitutive activity of GHSR1a A204E. It was recently shown that the WT GHSR1a exhibits high constitutive activity (15). To test whether the A204E mutant GHSR1a displays the same property, given its decreased cell-surface expression, we monitored the constitutive activity of the WT and the mutant GHSR1a as a function of their own cell-surface expression. To this end, we studied the signal transduction triggered by the WT or the mutant receptor transiently expressed in HEK293 cells grown in the absence of ligand, using a POU1F1-luciferase (POU1F1-Luc) reporter assay that reflects the cAMP-response element (CRE) pathway (16). Both the WT and the mutant receptors were tagged at their $\mathrm{N}$-terminus with an HA epitope, in order to assess their basal activity relative to their cell-surface expression. This experiment, shown in Figure 5A, disclosed the following features. Firstly, the cell-surface expression of GHSR1a A204E was severely impaired, close to $20 \%$ of that of the WT receptor, a result in agreement with our previous data (Figure 4A). Secondly, increasing the expression of the WT receptor was associated with an increase in the luciferase signal in a dosedependent manner (slope $\left.=33,103 ; r^{2}=0.97\right)$, in keeping with a previous study (15). In contrast, a similar experiment performed 
Table 2

Clinical and biological phenotypes of the children with short stature from families 1 and 2 before $\mathrm{GH}$ treatment, according to genotype

\begin{tabular}{lcccc} 
& \multicolumn{3}{c}{ Family 1 } & Family 2 \\
& II2 & II3 & II4 & II1 \\
Clinical data & & & & \\
Sex & $\mathrm{F}$ & $\mathrm{M}$ & $\mathrm{F}$ & $\mathrm{F}$ \\
Age (years) & 13.8 & 10.6 & 7.7 & 11.5 \\
Height $(\mathrm{cm})$ & 137 & 128 & 112 & 125 \\
SD for age & -3.7 & -2.0 & -2.2 & -3.2 \\
Weight $(\mathrm{kg})$ & 49 & 32 & 22 & 20 \\
BMI $\left(\mathrm{kg} / \mathrm{m}^{2}\right)$ & $26.2^{\mathrm{B}}$ & 19.7 & 17.4 & 12.8 \\
Puberty $($ Tanner stage) & $\mathrm{P} 5$ & $\mathrm{P} 1$ & $\mathrm{P} 1$ & $\mathrm{P} 1$ \\
GH treatment & Yes & Yes & No & Yes \\
Biological data & & & & \\
Basal IGF-1 $(\mathrm{ng} / \mathrm{ml})$ & 371 & 173 & 103 & 28 \\
GH peak $(\mu \mathrm{IU} / \mathrm{ml})^{\mathrm{C}}$ & $29 ; 48$ & $14 ; 9$ & 43 & $3 ; 5$ \\
Total ghrelin $(\mathrm{pg} / \mathrm{ml})^{\mathrm{D}}$ & 1,598 & 1,708 & 1,858 & 2,027 \\
Active ghrelin $(\mathrm{pg} / \mathrm{ml})^{\mathrm{E}}$ & 201 & 252 & 353 & 539 \\
Diagnosis & $\mathrm{ISS}$ & $\mathrm{IGHD}$ & $\mathrm{ISS}$ & $\mathrm{IGHD}$ \\
GHSR genotype & $\mathrm{M} / \mathrm{M}$ & $\mathrm{M} / \mathrm{N}$ & $\mathrm{M} / \mathrm{N}$ & $\mathrm{M} / \mathrm{N}$ \\
& & & &
\end{tabular}

AHeight SDs according to French reference values (38). BOverweight for age according to ref. 39. CNormal range $>20 \mu \mathrm{IU} / \mathrm{ml}$. DNormal range for prepubertal individuals: $336-3,320 \mathrm{pg} / \mathrm{ml}$. ENormal range for prepubertal individuals: $22-889 \mathrm{pg} / \mathrm{ml}$. FN and $\mathrm{M}$ represent the normal and the mutated A204E GHSR alleles, respectively.

with the A204E receptor showed a much lower increase in basal activity (Figure 5A). Taken together, these data, which reflect the degree of ligand-independent signaling activity of the GHSR1a, therefore, demonstrate that the A204E mutation leads to a loss of constitutive activity of the receptor, which results from both its intrinsic inability to signal constitutively and its decreased cell-surface expression.

To investigate the molecular mechanism underlying the dominant mode of inheritance of the short stature phenotype, we assayed transcription from the same POU1F1-Luc reporter gene in the presence of both WT and mutant GHSR proteins. As shown in Figure 5B, cotransfection of equal amounts of the plasmids encoding these 2 receptors did not inhibit the constitutive activity associated with the WT receptor, thereby suggesting the absence of a dominant-negative effect of the mutant A204E over the WT GHSR1a, at least in that system.

Maintained ghrelin-mediated signal transduction of GHSR1a A204E. To further characterize the biological properties of this particular mutant, we assessed its ability to activate the CRE pathway in response to different ligands. To this end, we incubated HEK293 cells expressing the WT or the mutated receptor in the presence of ghrelin or of [D-Arg $\left.1, \mathrm{D}-\mathrm{Phe}^{5}, \mathrm{D}-\operatorname{Tr}^{7,9}, \mathrm{Leu}^{11}\right]$-substance $\mathrm{P}$, a molecule that had been shown to act as an inverse agonist for the constitutive signaling of the human GHSR1a (15). The results confirmed that the basal activity of the mutant receptor - assayed in the absence of any ligand - was undetectable (Figure 6A). Most importantly, they also revealed that ghrelin was able to stimulate the CRE pathway not only through the WT receptor, but also through the mutant one, in spite of its decreased cell-surface expression; furthermore, the response to ghrelin with respect to basal conditions was even more pronounced for the mutant receptor than for the WT
GHSR (270\% and 130\%, respectively). Finally, whereas, as previously demonstrated (15), the GHSR inverse agonist decreased the basal activity of the WT receptor to background levels, this was not the case for the mutant GHSR1a, on which [D-Arg ${ }^{1}$, D-Phe ${ }^{5}, \mathrm{D}-\operatorname{Tr}^{7,9}$, Leu $\left.^{11}\right]$-substance $\mathrm{P}$ had no significant effect. Indeed, as shown in Figure 6A, this molecule did not change the basal activity of the mutant receptor, which remained within background levels.

To test whether the properties of the mutant GHSR depicted here are specific to the POU1F1/CRE pathway or affect other GHSR signaling cascades, we performed similar experiments using a serum-response element-Luc (SRE-Luc) reporter assay that reflects the recently described SRE pathway activated by the GHSR (17). As shown in Figure 6B, this system also revealed a lack of constitutive activity of the mutant GHSR, in spite of a clear response of this receptor to ghrelin. The response to ghrelin with respect to basal conditions was indeed of $1,300 \%$ and $170 \%$ for the mutant and the WT receptor, respectively. Overall, our data on GHSR signaling strongly suggest that the A204E mutation results in a loss of constitutive activity and in a high response to ghrelin, at least in the 2 pathways studied above.

At last, to check more directly the ghrelin response of the A204E mutant receptor (i.e., at the second-messenger level of this Gqcoupled receptor; ref. 2 ), we analyzed, by means of an appropriate fluorescent reporter, the $\mathrm{Ca}^{2+}$ response in HEK293 cell lines stably transfected with GHSR. As shown in Figure 7, dose-response curves indicated, first, that the A204E receptor displayed a potency similar to that of the WT receptor $\left(\mathrm{EC}_{50}=5.4 \mathrm{nM}\right.$ and $4.3 \mathrm{nM}$ for A204E and WT, respectively), and second, that the A204E receptor displayed an efficiency slightly higher than that of the WT receptor (maximum effect $=55 \%$ and $42 \%$, respectively).

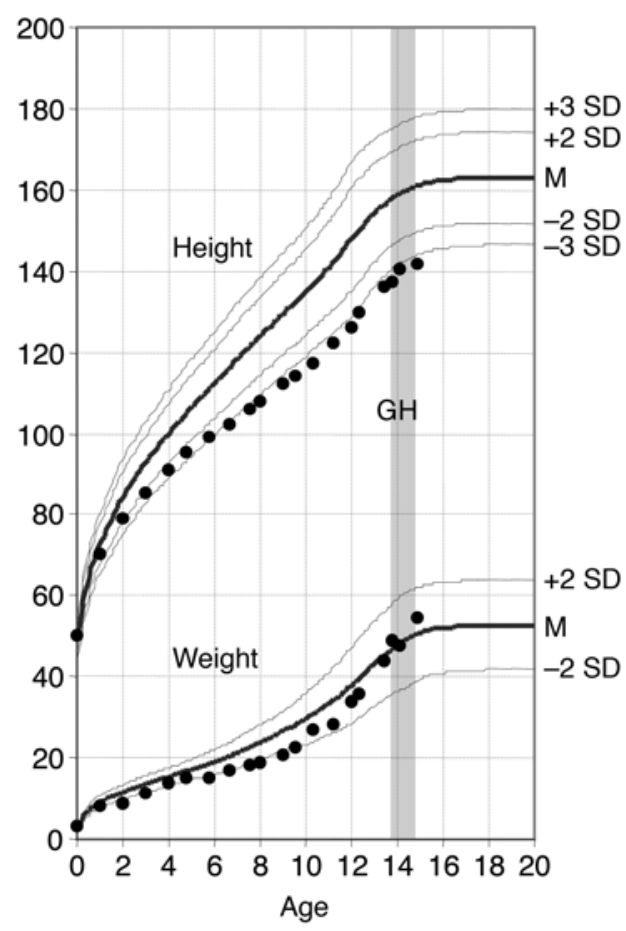

\section{Figure 3}

Height $(\mathrm{cm})$ and weight $(\mathrm{kg})$ curves of the proband (family 1, patient II2). The GH treatment is shown by the gray area. 
A

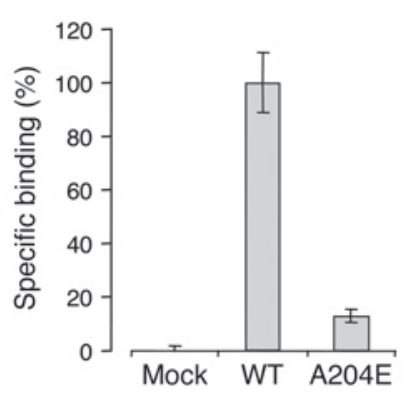

C
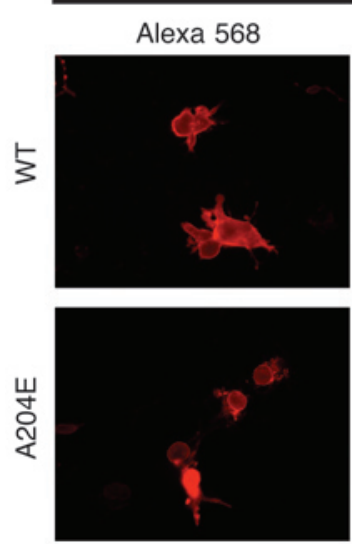

\section{B}
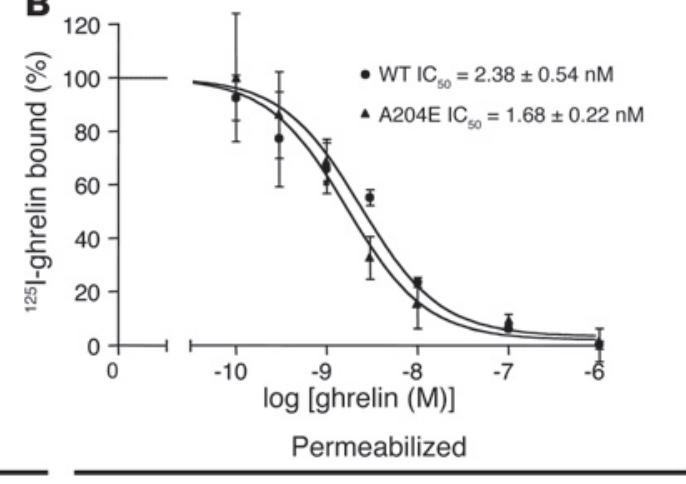

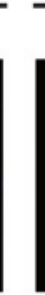

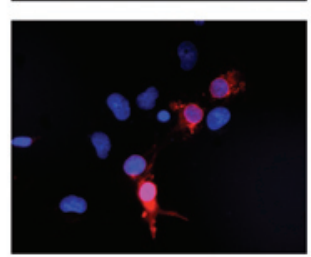

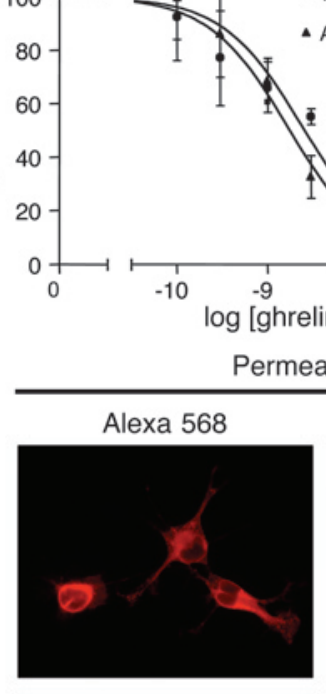

^ $\mathrm{A} 204 \mathrm{E} I \mathrm{IC}_{\mathrm{s0}}=1.68 \pm 0.22 \mathrm{nM}$

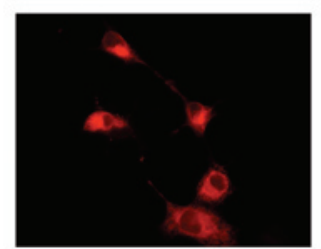

Figure 4

Expression of the A204E mutant GHSR1a at the cellular level. (A) Specific binding of 125 -ghrelin to HEK293 cells transiently transfected with empty vector (mock), or HA-GHSR-WT (WT) or HA-GHSR-A204E (A204E), as determined on whole cells. Transfected cells were incubated over 4 hours at $4^{\circ} \mathrm{C}$ with ${ }^{125} \mathrm{l}$-ghrelin $(30 \mathrm{pM})$. The specific binding, which represents the difference between total binding and nonspecific binding, is expressed as a percentage of the binding associated with the WT GHSR1a receptor. A representative experiment of 3 independent experiments (each performed in triplicate) is shown. (B) Displacement curves for 125l-ghrelin binding to whole HEK293 cells stably expressing the A204E mutant or the WT GHSR1a. The binding of $30 \mathrm{pM}$ of ${ }^{125}$ l-ghrelin was displaced by increasing concentrations of cold ghrelin. A representative experiment of 3 independent experiments (each performed in triplicate) is shown. (C) Immunolocalization of HA-tagged WT and A204E mutant receptors transiently expressed in COS-7 cells $(\times 40)$. The immunostaining of HA-tagged GHSR1a receptors was performed by means of an anti-HA monoclonal primary antibody incubated in the absence or in the presence of a permeabilizing reagent in order to visualize the receptors located at the cell surface or at both the cell surface and the intracellular level, respectively. Anti-HA mAb labeling was revealed by an Alexa Fluor 568 goat anti-mouse secondary antibody. Merge pictures show the anti-HA Alexa Fluor 568 staining (red) together with the staining of nuclei with DAPI (blue).

\section{Discussion}

In this study, we describe the molecular basis of a familial short stature, linked in 2 independent families to the same missense mutation in the GH secretagogue receptor (GHSR), which selectively impairs its constitutive activity, while preserving its ability to respond to ghrelin, its physiological ligand. This first description, to our knowledge, of a functionally significant GHSR mutation, which sheds new light on the current controversy about the physiological importance of normal secretagogue signaling in humans, unveils a novel and unusual pathogenic mechanism of growth failure.

Indeed, several lines of evidence support the involvement of the GHSR A204E mutation in the short stature phenotype. Firstly, in the 2 families of Moroccan origin in which short stature is transmitted over at least 2 generations, all the patients were shown to carry this mutation, whereas this defect was not found in an appropriate control population. Secondly, the
- WT IC $\mathrm{C}_{\mathrm{so}}=2.38 \pm 0.54 \mathrm{nM}$
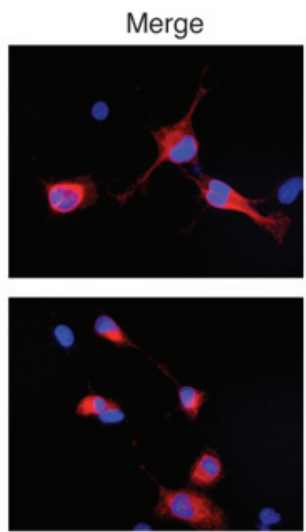

mutation predicts changes in the amino acid polarity and charge at a position invariant among species. Thirdly, we show that, while responding well to ghrelin, this particular GHSR mutant has lost its constitutive activity, a result that, in turn, points to the functional importance of this latter property recently attributed to the WT receptor, and whose clinical relevance was unknown (15).

Intrafamilial segregation analysis of the mutant GHSR allele and phenotype/genotype correlation studies disclose the following features associated with this mutation. As shown in Figure 2, the short stature segregates in a dominant manner. Although the height of individuals who carry the A204E mutation in the heterozygous state is clearly abnormal (from -2.7 SD to $-3.7 \mathrm{SD}$ ) in several cases (family 1-I1, family 1-I2, and family 2-II1), this is not always the case: 3 individuals (family 1-II3, family 1-II4, and family 2-I2) have a mild phenotype, while 3 others (family 1-II1, family 2-II2, and family 2-II3) have a height within the normal range. One patient (family 1-II2), who was born to a consanguineous union, was shown to carry the GHSR defect in the homozygous state; the fact that she had the most severe height reduction when compared with her heterozygous siblings is consistent with a semidominant mode of inheritance of the short stature phenotype. Altogether, these observations reflect an incomplete penetrance and a variable expressivity of the short stature phenotype, 2 common features of autosomal dominant traits; they also suggest the existence of other genetic and/or environmental factors that may contribute to the height of each individual.

Given the documented pharmacological effects of ghrelin on GH release $(5,6)$, it is particularly tempting to speculate that the short stature of these patients results from abnormal regulation of the GH/IGF-1 axis. In keeping with this hypothesis, 2 of the 4 children with short stature (i.e., family 1-II3 and family 2-II1) are indeed GHdeficient (IGHD), a feature that is not systematically associated with low IGF-1 values (family 1-II3). However, the 2 other children (family 1-II2 and family 1-II4) displayed a normal GH response to provocative tests and serum IGF-1 values within the normal range. 


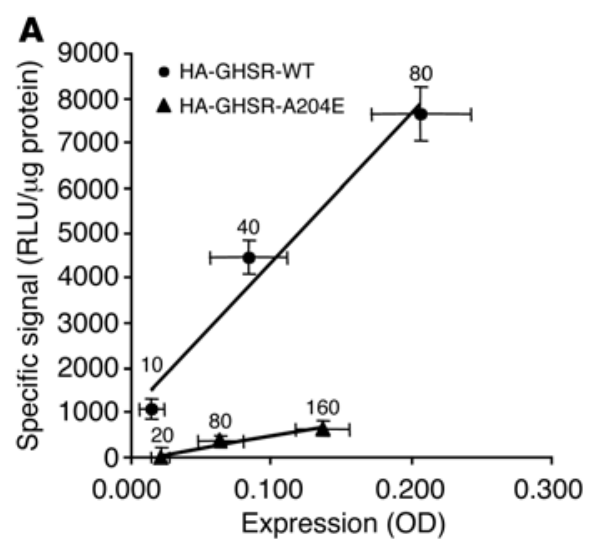

\section{Figure 5}

A204E GHSR1a agonist-independent signal transduction via the CRE pathway in transiently transfected HEK293 cells. (A) Constitutive activity of GHSR1a as a function of its own cell-surface expression. The constitutive activity associated with increasing concentrations of WT or A204E GHSR1a vectors was assessed in cotransfection experiments with an HA-tagged GHSR1a plasmid (HA-GHSR-WT or HAGHSR-A204E) and a CRE-containing reporter plasmid (pPOU1F1-Luc). The corresponding luminometric (RLU) signal was normalized to protein concentration (RLU/ $\mu \mathrm{g}$ proteins). The HA-GHSR1a cell-surface expression detected by means of an anti-HA epitope antibody (ELISA) is expressed in OD units. Signals associated with cells transfected with the mock plasmid have been subtracted, so that plotted signals represent specific RLU. Values are the mean \pm SD of 1 representative experiment performed in 4 replicates among 3 independent experiments. (B) Evaluation of a putative dominant-negative effect of the A204E mutant on the WT GHSR1a. Constitutive activity in cells expressing the GHSR1a WT, the GHSR1a A204E mutant, or both isoforms is expressed as a percentage of the basal activity of the WT GHSR1a receptor. Values are the mean \pm SD of 3 independent experiments, each performed in triplicate.
(11-14). Indeed, whereas the normal phenotype of ghrelin ${ }^{-/}$mice suggested that ghrelin itself does not seem to be critically required for growth $(12,14), \mathrm{ghsr}^{-1}$ mice display a modest but significant decrease in body weight and Igf-1 levels, compared with WT animals (13). Interestingly, transgenic rats expressing an antisense GHSR mRNA in the arcuate nucleus of the hypothalamus showed a more obvious phenotype (11). However, although both males and females were reported to be significantly smaller than controls, only females had biological alterations of the Gh/Igf- 1 axis, with a decrease in $G$ hecretion and plasma Igf-1 levels. Therefore, in spite of the heterogeneity of findings regarding the effect on the Gh/Igf-1 axis of ghrelin signaling disruption, several data - including those from the current study - support the debated view that ghrelin and the GH/IGF-1 axis are interrelated in a physiologically important way (19-21).
Nevertheless, one should keep in mind that the tests routinely used to establish the diagnosis of IGHD show considerable interindividual variations (18) and are far from being physiologically relevant, since they are based on the ability of the pituitary to release GH in response to high doses of various pharmacological stimuli. In such conditions, it is reasonable to assume that any subtle alteration in $\mathrm{GH}$ secretion could not be easily identified. Overall, the short stature identified in several patients with a GHSR mutation from 2 families, together with the abnormal findings on the GH/IGF-1 axis, therefore supports the hypothesis that GHSR signaling plays a significant role in controlling growth in the human, and that the underlying mechanism may involve a fine regulation of the somatotropic axis. In this regard, it is worth mentioning that the Gh/Igf- 1 axis of 4 different murine models carrying mutations expected to alter ghrelin signaling has been shown to be either normal or subnormal

\section{Figure 6}

Ligand-mediated signal transduction of the A204E GHSR1a in transiently transfected HEK293 cells. Cells expressing WT GHSR1a, GHSR1a A204E, or mock-transfected cells were incubated either in the absence or in the presence of an agonist (ghrelin at $10^{-6} \mathrm{M}$ ), or of an inverse agonist ([D-Arg ${ }^{1}, \mathrm{D}-\mathrm{Phe}^{5}, \mathrm{D}-\mathrm{Trp}^{7,9}$ Leu $\left.^{11}\right]$-substance $\mathrm{P}$, or SPA, at $\left.10^{-6} \mathrm{M}\right)$. The transcriptional activity in each condition (RLU/ug proteins) is expressed as a percentage of the basal activity of the WT GHSR1a receptor. Values are the mean \pm SD of 1 representative experiment performed in triplicate among 6 independent experiments. ${ }^{*} P<0.05$ and ${ }^{* *} P<0.01$ (ligand-induced signal versus basal level). (A) CRE-mediated transcriptional activity. Cells were cotransfected with $100 \mathrm{ng}$ of each expression plasmid and $250 \mathrm{ng}$ of the pPOU1F1-Luc reporter plasmid. (B) SRE-mediated transcriptional activity. Cells were cotransfected with $10 \mathrm{ng}$ of each expression plasmid and $500 \mathrm{ng}$ of the pSRE-Luc reporter plasmid.
In addition, it is important to bear in mind that all the mutations that have been introduced in mice or rats are expected to result in a complete loss of function of the targeted protein, i.e., ghrelin or its receptor. This is, however, obviously not the case for the mutation identified in these 2 families. Indeed, as shown by the study of different GHSR1a signaling cascades (i.e., CRE, SRE, and phospholipase C pathways), the GHSR A204E mutation, which has no major impact on the affinity of the receptor for ghrelin, does not prevent the ability of the GHSR1a to
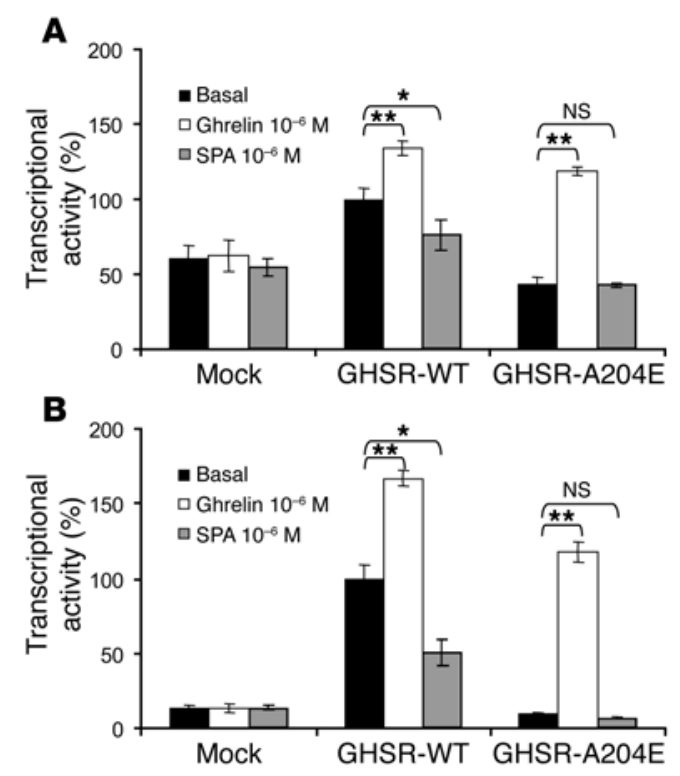


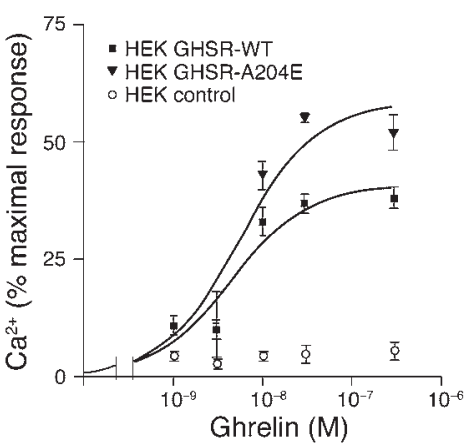

Figure 7

Calcium response to ghrelin of the WT and A204E GHSR1a receptors stably expressed in HEK293 cells. The HEK293 clones expressing the WT or the mutant receptor were selected on their similar GHSR1a expression pattern. GHSR1a-expressing cells and untransfected control cells were stimulated with increasing concentrations of ghrelin. The intracellular $\mathrm{Ca}^{2+}$ release was monitored by means of the Fluo-4 AM fluorescent probe. Each signal, which represents the mean of triplicates, is expressed as the percentage of total calcium release after ghrelin injection; total calcium release within a well corresponds to the peak obtained after cell lysis in the presence of $0.2 \%$ Triton. A representative experiment of 3 independent experiments (each performed in triplicate) is shown.

respond to ghrelin. This mutation is actually detrimental to the particularly high degree of constitutive activity of the GHSR1a (15), a property of a number of $G$ protein-coupled receptors (GPCRs) $(22,23)$. A cluster of aromatic residues located on the inner side of transmembrane segments VI and VII is critical for GHSR1a constitutive activity (17). Alanine 204, which is located within the second exoloop of the GHSR1a, is distant from the residues known to participate in the ligand-binding pocket (24), an observation that is in keeping with the absence of effect of the A204E mutation on the binding affinity of the receptor. However, alanine 204 is also located away from the cluster of aromatic residues located on the inner side of transmembrane segments VI and VII that is reported to be critical for GHSR constitutive activity (17), thereby raising the question of the mechanism by which the A204E mutation impairs the constitutive activity of the receptor. Although, as mentioned above, the A204E mutation has no effect on ghrelin binding, we cannot rule out the possibility that it induces selective conformational modifications that, in turn, impair constitutive activity. Altogether, these observations are consistent with a rather localized effect of the A204E mutation that selectively impairs both the cell-surface expression and the constitutive activity of the receptor, without altering its ability to bind ghrelin and subsequently activate at least the downstream signaling cascades studied above.

The mechanism by which this GHSR mutation exerts its deleterious effect is, therefore, quite unusual. Indeed, the mutations commonly found in other GPCRs result either in a gain of function (25), or in a partial or complete loss of function explained by the inability of the mutant receptor to be activated by its physiological ligand acting as an agonist (26-28). Such loss-of-function mutations have been identified in numerous receptors, including the melanocortin-4 receptor (MC4R), in which they have been shown to be associated with obesity (29, 30). As for the implication of MC4R in obesity, just recently,
Vaisse and colleagues showed that a subset of mutations located in the extracellular domain of the receptor selectively impair its constitutive activity, without affecting the signal transduction elicited by its ligand $\alpha$-melanocyte-stimulating hormone (31). From a more general viewpoint, this latter situation, which is, therefore, reminiscent of our data, underscores the functional importance of the constitutive activity of GPCRs in vivo, while raising the possibility that, depending on the involved GPCR, its selective loss could represent a molecular mechanism underlying other disease phenotypes.

Besides the short stature, it is noteworthy that several members of family 1 are overweight or obese. The same A204E mutation has recently been reported in one young obese patient within the framework of a study designed to screen the GHSR gene for sequence variations in a large population sample consisting of patients who presented with obesity or short stature (32). However, according to this study, no conclusive evidence for the involvement of the GHSR in body weight regulation or in short stature could be provided. Such an obese phenotype is rather unexpected, given both the pharmacological effects of ghrelin on food intake and fat deposition $(7-9,33)$ and the phenotypic studies of genetically modified murine models $(11,13)$. One should also keep in mind that 1 of these patients (family 1-II3) is clearly GH-deficient - a condition known to be associated with increased adiposity while he displays only a trend to overweight; on the other hand, no GH deficiency could be documented in patient II2, who displays a clear overweight. These observations, together with the fact that the nonobese patient II 1 from family 2 is GH-deficient, strongly argue against the hypothesis that the increased adiposity of several patients with the A204E GHSR allele results from GH deficiency. Therefore, these data raise the question of whether the overweight observed in several members of family 1 reflects a direct effect of this particular GHSR mutation on energy homeostasis through a mechanism that is still to be elucidated, or a consequence of other genetic and/or environmental factors involved in energy homeostasis $(34,35)$. The recent report of genetic linkage and association of GHSR with obesity in the human (36) would rather support the former hypothesis; however, given the normal weight of patients from family 2 , the latter cannot be excluded.

This functionally significant GHSR mutation - the first to our knowledge - found in patients with a short stature, therefore, identifies a new etiology of growth failure in humans. The current data also demonstrate the critical importance of the constitutive activity of the GHSR, which has been described recently by in vitro means and, as shown here, represents a key physiological component of the GHSR axis.

\section{Methods}

Subjects. Two groups of patients presenting with IGHD $(n=51)$ or with ISS $(n=41)$ were studied. The ISS group is composed of patients followed in France; as for patients with IGHD, 26 of them are followed in France, whereas 25 are followed in Morocco. The diagnosis of ISS was made according to established criteria (37), whereas that of IGHD was based on GH peak values below $20 \mu \mathrm{IU} / \mathrm{ml}$ in response to 2 pharmacological stimulation tests. Written informed consent was obtained from individuals participating in this study, which was approved by the Comité Consultatif de Protection de Personnes dans la Recherche Biomédicale de Créteil-Henri Mondor (Créteil, France).

GHSR mutation analysis. The 2 GHSR coding exons and their flanking intronic regions were amplified by PCR from genomic DNA extracted from whole blood. The following primer sets were used: P1 (5'-CAGTCAC- 
GTCCCAGAGCCT-3') and P3 (5'-CACAGGGAGAGGATAGGACC-3') for exon 1, and P2 (5'-CTGTGACATTTCTTGAGCTGAC-3') and P4 (5'GTTCTGCTGTGCTATGTCTTCC-3') for exon 2. Amplified products were subsequently directly sequenced on a PerkinElmer 3100 DNA sequencer (Applied Biosystems). Segregation analysis of the identified GHSR A204E point mutation within affected families and screening for this sequence variation in a control population were accomplished by $\mathrm{MnlI}$ digestion of the PCR products spanning the mutation site and generated with primers P5 (5'-GCCCATCTTCGTGCTAGTCG-3') and P6 (5'-AAGATGCTGGACACCCACAC- $3^{\prime}$ ). Digestion products were analyzed by electrophoresis on a $6 \%$ acrylamide gel stained with ethidium bromide.

GHSR1a plasmid constructs. The WT GHSR 1a isoform (1) cDNA was amplified by PCR using human adult pituitary cDNA as template (Clontech Laboratories Inc.) and the couple of primers P1/P2. The resulting PCR product was subsequently cloned into the pcDNA3.1/V5-His Topo TA expression vector (Invitrogen Corp.) to generate the WT cDNA GHSR plasmid (GHSR-WT). This plasmid was subjected to site-directed mutagenesis by the QuikChange kit (Stratagene) by means of oligonucleotides designed to introduce an HA epitope (YPYDVPDYA) at the N-terminus of the receptor (plasmid HA-GHSR-WT). Finally, these 2 WT GHSR plasmids were subjected to site-directed mutagenesis by means of oligonucleotides designed to introduce the C-to-A substitution found in the patients; the resulting plasmids were designated GHSR-A204E and HA-GHSR-A204E.

HEK293 GHSR1a stable clones. To generate clones stably expressing the WT GHSR1a or the A204E mutant GHSR1a, HEK293 cells were transfected with the GHSR-WT or the GHSR-A204E plasmid by the Lipofectamine method (Invitrogen Corp.); individual clones were selected upon geneticin treatment $(500 \mu \mathrm{g} / \mathrm{ml})$. Among these clones selected on the basis of their GHSR transcript expression pattern - as determined by Northern blotting -2 were retained for further functional studies.

${ }^{125}$ I-ghrelin binding studies. Whole cells expressing the GHSR (transiently transfected COS-7 or HEK293 cells, or HEK293 cells stably expressing the receptor) were incubated with $30 \mathrm{pM}\left[{ }^{125} \mathrm{I}-\mathrm{His}^{9}\right]$ ghrelin $1-28$ at 2,000 Ci/ $\mathrm{mmol}$ (Amersham Biosciences) for 1 hour at $37^{\circ} \mathrm{C}$ or over 4 hours at $4^{\circ} \mathrm{C}$ within $1 \%$ BSA binding buffer. The specific binding ( $>80 \%$ of total binding) equaled the difference between total and nonspecific binding obtained in the presence of $50 \mathrm{nM}$ of cold ghrelin. The estimate of the $\mathrm{IC}_{50}$ values was performed using Prism 3 software (GraphPad Software).

Immunocytochemistry. COS-7 transfectants expressing HA-GHSR-WT, HA-GHSR-A204E, or mock (pcDNA3.1 empty vector) were plated onto glass coverslips and fixed in $4 \%(\mathrm{vol} / \mathrm{vol})$ paraformaldehyde/PBS and then washed in PBS. Intact cells were washed with $1 \% \mathrm{BSA} / \mathrm{PBS}$ prior to incubation with a 1:100 dilution of anti-HA $6 \mathrm{E} 2 \mathrm{mAb}$ (Cell Signaling Technology) for 1 hour at room temperature followed by a 30 -minute washing step in $1 \%$ BSA/PBS. Cells were then incubated with a 1:500 dilution of secondary Alexa Fluor 568 goat anti-mouse antibody (Invitrogen Corp.) for 1 hour at room temperature and subsequently washed with $1 \% \mathrm{BSA} / \mathrm{PBS}$ before mounting. To work on permeabilized cells, the above-mentioned steps were performed in $0.1 \%$ Triton X-100/PBS. Finally, intact and permeabilized stained cells were examined with a Leica DMR fluorescence microscope.

GHSR1a transcriptional activity. To study the transcriptional activity of GHSR1a, HEK293 cells (grown in the absence or the presence of a ligand) transiently expressing the A204E mutant or the WT GHSR (with or without an HA tag) were transfected with a GHSR promoter signaling target coupled to a luciferase reporter gene. The 2 following targets were used in these assays: (a) a CRE-containing 837-bp POU1F1 promoter sequence (16) inserted upstream from the $L u c$ coding sequence within the pGL3 basic vector (Promega) - the resulting plasmid was designated pPOU1F1-Luc; and (b) an SRE consensus from a commercially available vector named pSRE-Luc (Stratagene). Various concentrations of the GHSR or mock expression plasmids were cotransfected alternatively with $250 \mathrm{ng} /$ well of pPOU1F1-Luc or $500 \mathrm{ng} /$ well of pSRE-Luc. These cotransfection experiments were performed in triplicate in 12-well plates according to the Lipofectamine method (Invitrogen Corp.). Luminescence was measured using the Lumat LB 9507 luminometer (Berthold Technologies) by means of the luciferase assay system (Promega), and the data were normalized to protein concentration.

To compare the constitutive activity of the A204E mutant with that of the WT GHSR1a, each construct was expressed at increasing amounts (range 10-160 ng plasmid per well). The 2 constructs were also expressed simultaneously, in order to study the effect of the A204E mutant GHSR1a on the constitutive activity associated with the WT GHSR1a. To compare the ligand-mediated response of the A204E mutant with that of the WT GHSR1a, 24 hours after transfection, transfected cells were incubated in the presence either of ghrelin $\left(10^{-6} \mathrm{M}\right)$ (NeoMPS) or of a GHSR1a-inverse agonist ([D-Arg ${ }^{1}$, D-Phe ${ }^{5}$, D-Trp ${ }^{7,9}$, Leu $\left.^{11}\right]$-substance P) $\left(10^{-6} \mathrm{M}\right)$ (NeoMPS), or in the absence of any ligand (basal conditions). These experiments were performed for 6 hours at $37^{\circ} \mathrm{C}$ in DMEM with $2.5 \%$ FCS.

GHSR1 a cell-surface expression (ELISA). To quantify the cell-surface expression of GHSR1a, additional wells of HEK293 cells transiently cotransfected with each of the HA-tagged GHSR1a expression plasmids and PPOU1F1Luc (see above) were processed as follows. Transfectants were fixed in $4 \%$ paraformaldehyde for 10 minutes; washed; saturated in PBS, $5 \%$ goat serum, and $1 \% \mathrm{BSA}$ for 30 minutes at $4{ }^{\circ} \mathrm{C}$; and incubated over 1 hour at room temperature with an HRP-conjugated anti-HA goat polyclonal antibody (Bethyl Laboratories Inc.). After 3 PBS washes, the immunoreactivity was revealed by 30 minutes' incubation with $o$-phenylenediamine solution (Sigma-Aldrich); the reaction was stopped by addition of sulfuric acid. Each sample was transferred into a 96-well plate for quantification at $490 \mathrm{nM}$ in a microplate spectrophotometer.

Calcium mobilization assays. To evaluate the GHSR1a ligand-mediated activation via the phospholipase $\mathrm{C}$ pathway, the intracellular $\mathrm{Ca}^{2+}$ release was measured by means of a $\mathrm{Ca}^{2+}$-sensitive fluorescent reporter (Fluo-4 AM; Invitrogen Corp.), on HEK293 clones stably overexpressing the A204E mutant or the WT GHSR1a. Stable clones and control HEK cells were incubated with $4 \mu \mathrm{M}$ of Fluo- $4 \mathrm{AM}$ for 1 hour at $37^{\circ} \mathrm{C}$ in loading buffer with $2 \mathrm{mM}$ of probenecid. Once washed, loaded cells distributed within 96well black microplaques were stimulated with increasing concentrations of ghrelin. For each well, fluorescence (excitation $494 \mathrm{~nm}$, emission at 516 $\mathrm{nm}$ ) was recorded on a microplaque fluorometer to determine 3 values: base line, ligand peak, and total calcium release. Results are expressed as the percentage of total calcium release after ligand injection; total calcium release within a well corresponds to the peak obtained after cell lysis by means of $0.2 \%$ Triton injection. All values are the mean of triplicates.

Ghrelin assays. Plasma ghrelin isoforms were assayed by enzyme immunoassay using 2 rabbit polyclonal antibodies raised against the 15-28 C-terminus fragment for total ghrelin (gift from C. Tomasetto, INSERM, Strasbourg, France) and the $n$-octanoylated 1-11 N-terminus fragment for acylated ghrelin (gift from M. Kojima, Kurume University, Fukuoka, Japan). Human $n$-octanoyled ghrelin was used as standard (Phoenix Pharmaceuticals) and coupled to acetylcholinesterase as tracer (SPI-BIO). Antibodies were used at a final dilution of $1: 6.10^{5}$ for $\mathrm{C}$-terminus and $1: 10^{7}$ for N-terminus. Their limit of detection was $50 \mathrm{pg} / \mathrm{ml}(50-4,000)$ and $10 \mathrm{pg} / \mathrm{ml}(10-400)$, respectively. Out of 8 consecutive assays, intra-assay and interassay variabilities were $4 \%$ and $7 \%$ for C-terminus assay and $5 \%$ and $6 \%$ for $\mathrm{N}$-terminus assay, respectively.

Statistics. Data are expressed as mean $\pm \mathrm{SD}$. Comparison of means was carried out using 2-tailed Student's $t$ test. $P<0.05$ was considered statistically significant. In immunocytochemistry experiments, comparisons of the number of stained cells were performed by means of $\chi^{2}$ test. 


\section{Acknowledgments}

We are indebted to the children and parents of these families for agreeing to participate in this study, and to Philippe Duquesnoy, Marie-Laure Sobrier, and Kalotina Machinis for fruitful discussions. This work was supported by INSERM and grants from the Assistance Publique - Hôpitaux de Paris (CRC96085, PHRC 2003 no. 25/2003) and INSERM/Centre National de la Recherche Scientifique et Technique (08/05-06). M. Legendre was the recipient of a fellowship from the Fondation pour la Recherche Médicale.
Received for publication April 8, 2005, and accepted in revised form December 6, 2005.

Address correspondence to: Jacques Pantel, INSERM, U654, 51 avenue du Maréchal de Lattre-de-Tassigny, 94010 Créteil Cedex, France. Phone: 33-149-81-28-56; Fax: 33-148-99-33-45; E-mail: jacques.pantel@im3.inserm.fr.

Jacques Pantel and Marie Legendre contributed equally to this work.
1. Howard, A.D., et al. 1996. A receptor in pituitary and hypothalamus that functions in growth hormone release. Science. 273:974-977.

2. Smith, R.G., et al. 1997. Peptidomimetic regulation of growth hormone secretion. Endocr. Rev. 18:621-645.

3. Kojima, M., et al. 1999. Ghrelin is a growth-hormone-releasing acylated peptide from stomach. Nature. 402:656-660.

4. Cummings, D.E., et al. 2001. A preprandial rise in plasma ghrelin levels suggests a role in meal initiation in humans. Diabetes. 50:1714-1719.

5. Takaya, K., et al. 2000. Ghrelin strongly stimulates growth hormone release in humans.J. Clin. Endocrinol. Metab. 85:4908-4911.

6. Arvat, E., et al. 2001. Endocrine activities of ghrelin, a natural growth hormone secretagogue (GHS), in humans: comparison and interactions with hexarelin, a nonnatural peptidyl GHS, and GH-releasing hormone. J. Clin. Endocrinol. Metab. 86:1169-1174.

7. Tschop, M., Smiley, D.L., and Heiman, M.L. 2000. Ghrelin induces adiposity in rodents. Nature. 407:908-913.

8. Wren, A.M., et al. 2000. The novel hypothalamic peptide ghrelin stimulates food intake and growth hormone secretion. Endocrinology. 141:4325-4328.

9. Nakazato, M., et al. 2001. A role for ghrelin in the central regulation of feeding. Nature. 409:194-198.

10. Wren, A.M., et al. 2001. Ghrelin enhances appetite and increases food intake in humans. J. Clin. Endocrinol. Metab. 86:5992-5995.

11. Shuto, Y., et al. 2002. Hypothalamic growth hormone secretagogue receptor regulates growth hormone secretion, feeding, and adiposity. J. Clin. Invest. 109:1429-1436. doi:10.1172/JCI200213300.

12. Sun, Y., Ahmed, S., and Smith, R.G. 2003. Deletion of ghrelin impairs neither growth nor appetite. Mol. Cell. Biol. 23:7973-7981.

13. Sun, Y., Wang, P., Zheng, H., and Smith, R.G. 2004 Ghrelin stimulation of growth hormone release and appetite is mediated through the growth hormone secretagogue receptor. Proc. Natl. Acad. Sci. U. S. A. 101:4679-4684.

14. Wortley, K.E., et al. 2004. Genetic deletion of ghrelin does not decrease food intake but influences metabolic fuel preference. Proc. Natl. Acad. Sci. U. S. A. 101:8227-8232.

15. Holst, B., Cygankiewicz, A., Jensen, T.H., Ankersen, M., and Schwartz, T.W. 2003. High constitutive signaling of the ghrelin receptor: identification of a potent inverse agonist. Mol. Endocrinol. 17:2201-2210.

16. Garcia, A., Alvarez, C.V., Smith, R.G., and Dieguez, C. 2001. Regulation of Pit-1 expression by ghrelin and GHRP-6 through the GH secretagogue receptor. Mol. Endocrinol. 15:1484-1495.

17. Holst, B., et al. 2004. Common structural basis for constitutive activity of the ghrelin receptor family. J. Biol. Chem. 279:53806-53817.

18. Ranke, M.B., and Haber, P. 1996. Growth hormone stimulation tests. In Diagnostics of endocrine function in children and adolescents. M.B. Ranke and N. Albers, editors. Johann Ambrosius Barth Verlag. Heidelberg, Germany. 134-148.

19. Cappiello, V., et al. 2002. Circulating ghrelin levels in basal conditions and during glucose tolerance test in acromegalic patients. Eur. J. Endocrinol. 147:189-194.

20. Freda, P.U., Reyes, C.M., Conwell, I.M., Sundeen, R.E., and Wardlaw, S.L. 2003. Serum ghrelin levels in acromegaly: effects of surgical and long-acting octreotide therapy. J. Clin. Endocrinol. Metab. 88:2037-2044

21. Eden Engstrom, B., Burman, P., Holdstock, C., and Karlsson, F.A. 2003. Effects of growth hormone $(\mathrm{GH})$ on ghrelin, leptin, and adiponectin in GH-deficient patients. J. Clin. Endocrinol. Metab. 88:5193-5198.

22. Morisset, S., et al. 2000. High constitutive activity of native $\mathrm{H} 3$ receptors regulates histamine neurons in brain. Nature. 408:860-864.

23. Seifert, R., and Wenzel-Seifert, K. 2002. Constitutive activity of G-protein-coupled receptors: cause of disease and common property of wild-type receptors. Naunyn Schmiedebergs Arch. Pharmacol. 366:381-416.

24. Feighner, S.D., et al. 1998. Structural requirements for the activation of the human growth hormone secretagogue receptor by peptide and nonpeptide secretagogues. Mol. Endocrinol. 12:137-145.

25. Parnot, C., Miserey-Lenkei, S., Bardin, S., Corvol, P., and Clauser, E. 2002. Lessons from constitutively active mutants of $G$ protein-coupled receptors. Trends Endocrinol. Metab. 13:336-343.

26. Sunthornthepvarakui, T., Gottschalk, M.E., Hayashi, Y., and Refetoff, S. 1995. Brief report: resistance to thyrotropin caused by mutations in the thyrotropin-receptor gene. N. Engl. J. Med. 332:155-160.

27. Kremer, H., et al. 1995. Male pseudohermaphro- ditism due to a homozygous missense mutation of the luteinizing hormone receptor gene. Nat. Genet. 9:160-164.

28. Aittomaki, K., et al. 1995. Mutation in the follicle-stimulating hormone receptor gene causes hereditary hypergonadotropic ovarian failure. Cell. 82:959-968.

29. Vaisse, C., et al. 2000. Melanocortin-4 receptor mutations are a frequent and heterogeneous cause of morbid obesity. J. Clin. Invest. 106:253-262.

30. Farooqi, I.S., et al. 2000. Dominant and recessive inheritance of morbid obesity associated with melanocortin 4 receptor deficiency. J. Clin. Invest. 106:271-279.

31. Srinivasan, S., et al. 2004. Constitutive activity of the melanocortin- 4 receptor is maintained by its $\mathrm{N}$-terminal domain and plays a role in energy homeostasis in humans. J. Clin. Invest. 114:1158-1164. doi:10.1172/JCI200421927.

32. Wang, H.J., et al. 2004. Ghrelin receptor gene: identification of several sequence variants in extremely obese children and adolescents, healthy normalweight and underweight students, and children with short normal stature. J. Clin. Endocrinol. Metab. 89:157-162.

33. Holst, B., and Schwartz, T.W. 2004. Constitutive ghrelin receptor activity as a signaling set-point in appetite regulation. Trends Pharmacol. Sci. 25:113-117.

34. Barsh, G.S., Farooqi, I.S., and O'Rahilly, S. 2000. Genetics of body-weight regulation. Nature. 404:644-651.

35. Cummings, D.E., and Schwartz, M.W. 2003. Genetics and pathophysiology of human obesity. Annu. Rev. Med. 54:453-471.

36. Baessler, A., et al. 2005. Genetic linkage and association of the growth hormone secretagogue receptor (ghrelin receptor) gene in human obesity. Diabetes. 54:259-267.

37. Ranke, M.B. 1996. Towards a consensus on the definition of idiopathic short stature. Horm. Res. 45(Suppl. 2):64-66.

38. Sempé, M., Pedron, G., and Roy-Pernot, M.-P. 1979. Auxologie: methode et séquences. Laboratoire Theraplix. Paris, France. 205 pp.

39. Cole, T.J., Bellizzi, M.C., Flegal, K.M., and Dietz, W.H. 2000. Establishing a standard definition for child overweight and obesity worldwide: international survey. BMJ. 320:1240-1243. 